\title{
Medicamentos Vencidos ou em Desuso e Riscos Ambientais no Município de Terenos, Mato Grosso do Sul
}

\section{Expired or Disused Drugs and Environmental Risks in the Municipality of Terenos, Mato Grosso do Sul}

\author{
Gislaine Tonet ${ }^{\mathrm{a}}$; Ademir Kleber Morbeck de Oliveira ${ }^{\mathrm{a}}$; Carla Leticia Gediel Rivero-Wendt*a; Giselle Marques de \\ Araújo $^{\text {a }}$ Ingryd Khristina de Brito ${ }^{a}$; Amanda Rodrigues Ganassin ${ }^{\mathrm{a}}$; Rosemary Matias ${ }^{\mathrm{a}}$ \\ aUniversidade Anhanguera-Uniderp, Programa de Pós-Graduação Stricto Sensu em Meio Ambiente e Desenvolvimento Regional. MS, Brasil. \\ *E-mail: leticiagediel@gmail.com
}

\begin{abstract}
Resumo
No Brasil, o descarte de medicamentos e de insumos farmacêuticos de forma inadequada gera em torno de 10 e 28 mil toneladas, contribuindo para um problema de saúde pública e ambiental. Visando mitigar este cenário, o artigo tem como objetivo a caracterização e discussão dos potenciais impactos ambientais do descarte de medicamentos e/ou insumos farmacêuticos no lixo comum da cidade de Terenos, Mato Grosso do Sul (MS), a partir de coleta de quantificação realizada durante 2015 e 2017. A metodologia do estudo partiu de coleta em Unidade de Tratamento de Resíduos (UTR), com separação e quantificação dos medicamentos encontrados no lixo comum do município investigado. Foram coletados 80 tipos de fármacos que somaram um depósito total de cerca de $490 \mathrm{~g}$ a cada 15 dias, em projeção de descarte médio de 1,69 g ao ano de medicamentos em lixo comum por munícipe. Foram encontrados fármacos de alta toxidade (hormônios, antidepressivos e anti-helmínticos) que, embora fora das categorias principais de descarte, possuem potencial elevado de comprometimento ambiental. Conclui-se que o problema local do descarte de medicamentos em lixo comum é severo no município avaliado, no entanto, medidas mitigativas podem ser tomadas, tais como: a educação da população e a oferta de unidades para descarte preferencial nas Unidades Básicas de Saúde (UBS).
\end{abstract}

Palavras-chave: Resíduos Sólidos. Poluição Ambiental. Unidade de Tratamento de Resíduos.

\begin{abstract}
In Brazil, medicines and pharmaceutical inputs discarded inappropriately generates around 10 and 28 thousand tons, contributing to a problem in public and environmental health. Aiming to contribute to the deconstruction of this scenario, the aim of this study was characterize and discuss the environmental impacts of the medicines disposal and / or pharmaceutical input in the common waste of Terenos, Mato Grosso do Sul (MS), from collections to perform quantification and carried out during 2015 and 2017. The methodology started in the Waste Treatment Unit (WTU), separating, and quantifying the drugs found in the municipal waste. Eighty types of drugs were collected, accumulated a total of $490 \mathrm{~g}$ every 15 days, regarding the annual accumulation per citizen of $1.69 \mathrm{~g}$ of medicines in the common garbage. High toxicity drugs (hormones, antidepressants and anthelmintics) have been found, despite being out of the main disposal categories, have a high potential for environmental damage. It is concluded that the local problem of medicines disposal in common garbage is severe in Terenos, however, mitigative measures can be taken, such as the population environmental education and offering selective collect trash cans in Basic Health Units (BHU).
\end{abstract}

Keywords: Solid Waste. Environmental Pollution. Drugs. Waste Treatment Plant.

\section{Introdução}

A elevação do consumo de medicamentos ocorrida nas duas últimas décadas foi justificada por Alvarenga e Nicoletti (2010) e Santos e Ferreira (2012) como resultado da automedicação, do avanço da doenças crônicas e crônicodegenerativas, do fomento da mídia e do crescimento da indústria farmacêutica.

Apensar do elevado consumo diário de medicamentos pela população mundial, Fernandes e Petrovick (2004) observam que há difusa falta de orientação sobre o descarte desses produtos e o trato com a demanda específica de resíduos. Trata-se de um problema grave e capaz de atingir a saúde coletiva, pois os medicamentos não raros contém substâncias capazes de atingir a saúde ambiental e humana (ALVARENGA; NICOLETTI, 2010).

O mais comum é o descarte dos medicamentos no lixo doméstico, que leva sustâncias de alta interferência ambiental para espaços em que sua contenção e manejo não são devidamente previstos. Com isso, o solo, a fauna e a flora são expostos à ação de substâncias que podem alterar drasticamente o seu equilíbrio (EICKHOFF; HEINECK; SEIXAS, 2009). Alguns dos principais efeitos humanos sentidos são a resistência bacteriana (GHISELLI; JARDIM, 2007), que acarreta maior demanda de medicações e a nulidade ou baixa eficácia de muitos tratamentos.

O descarte inadequado de medicamentos, vencidos ou não e de suas embalagens totalmente consumidas ou com sobras, traz danos ambientais e à saúde pública. Mesmo o descarte eventual de alguns desses produtos, conforme as substâncias em deposição, pode ter como consequência impactos ambientais proeminentes, capazes de afetar o ambiente e gerar risco para a saúde de crianças ou pessoas carentes que possam reutilizá-los (FLAHERTY; DODSON, 2005; BOUND; 
VOULVOULIS, 2006).

O Brasil está entre os oito maiores consumidores mundiais de medicamentos. Atualmente, há cerca de 550 indústrias farmacêuticas, 79 mil drogarias e 7,1 farmácias de manipulação, 8,2 farmácias públicas e 5,4 mil hospitalares. Anualmente, a venda de medicamentos gera receita entre $\mathrm{R} \$$ 20,6 - R\$ 30,2 bilhões e segue crescente (ALVARENGA; NICOLETTI, 2010; SANTOS; FERREIRA, 2012).

$\mathrm{O}$ alto consumo de medicamentos é resultado de uma rede de fatores, que envolve a automedicação, o incentivo da mídia, o fácil acesso aos fármacos e os equívocos posológicos. Contribuem, ainda, as mudanças ou a descontinuidade do tratamento e as amostras-grátis da indústria farmacêutica (ALVARENGA; NICOLETTI, 2010; FERNANDES; PETROVICK, 2004).

Há ainda a colaboração da práxis de atendimento: entre $50 \%-70 \%$ das consultas geram prescrição de medicamentos e $50 \%$ dos pacientes os adquirem para apenas um dia de uso (AQUINO, 2008).

É uma prática que repercute, de formas diversas, entre as quais economicamente: países em desenvolvimento comprometem até $70 \%$ de suas verbas para saúde na aquisição de medicamentos (em países desenvolvidos, até $15 \%$ ) e entre 15\%-20\% das verbas de hospitais são aplicadas no cuidado de efeitos adversos do mau uso de fármacos (AQUINO, 2008).

Outro resultado do consumo crescente é o volume de embalagens e sobras. Há um acúmulo de medicamentos enquanto a maioria dos brasileiros desconhece os riscos desse comportamento e do descarte indevido de fármacos indesejados. Em razão da falta de informação do consumidor final, cerca de $20 \%$ dos medicamentos vencidos ou não terminam descartados em rede de esgoto ou lixo doméstico comum (ALVARENGA; NICOLETTI, 2010; BUENO; WEBER; OLIVEIRA, 2009; FALQUETO; KLIGERMAN; FACCHETTI ASSUMPÇÃO, 2010; FERNANDES; PETROVICK, 2004; SANTOS; FERREIRA, 2012).

Os medicamentos são resíduos do Grupo B, substâncias químicas capazes de causar risco à saúde pública ou ambiente, conforme as suas características de inflamabilidade, toxidade, reatividade e/ou corrosividade (ALVARENGA; NICOLETTI, 2010).

No Brasil, o gerenciamento de resíduos sólidos de saúde é regido pelas Resoluções Conama $n^{\circ}$ 358/2005 (BRASIL, 2005) e RDC ANVISA no 306/2004 (BRASIL, 2004), que dispõem sobre o gerenciamento externo e interno dos resíduos de serviços de saúde e atribui aos estabelecimentos de saúde o gerenciamento dos resíduos, da geração à deposição final.

Para as indústrias farmacêuticas, o processo de fabricação de medicamentos é normatizado pela RDC n. 210/2003, que trata das "Boas Práticas de Fabricação". A normativa orienta o tratamento de efluentes líquidos e emissões gasosas, antes que sejam lançados ao ambiente, abordando também como os resíduos sólidos devem ser tratados e destinados para a proteção ambiental e de saúde. Da mesma forma, é persistentemente cobrado dessas empresas que tenham o seu licenciamento ambiental atualizado, para controle de suas práticas e acompanhamento (FALQUETO; KLIGERMAN; ASSUMPÇÃO, 2010).

Prosseguem Falqueto, Kligerman e Assumpção (2010) relatando que distribuidores, farmácias, drogarias e hospitais se enquadram no escopo da RDC no 306/2004, expedida pela Agência Nacional de Vigilância Sanitária, que regula nacionalmente as disposições sobre o gerenciamento dos serviços de saúde. O descarte de medicamentos e a gestão de uso deve ser orientada pela Resolução CONAMA ${ }^{\circ}$ 358/2005, voltada ao tratamento e também disposição final dos resíduos sólidos, que são utilizados na prestação dos atendimentos de saúde, com a exigência de programa próprio para esse fim e licenciamento ambiental adequado para as medidas ligadas a sua ação no descarte e tratamento desses detritos (quando realizam).

Ainda, os mesmos autores observam que tipos específicos de medicamentos com alto impacto ambiental, como é o caso de hormônios e imunomoduladores, por exemplo, ou ainda insumos farmacêuticos sujeitos a controle especial, conforme Portaria $n^{\circ} 344 / 9825$, devem receber tratamento específico e orientado ao risco que apresentam em sua disposição, como colocação em sistemas próprios licenciados ou ainda em aterros de resíduos perigosos.

Tanto os objetos da Lei $n^{\circ} 11.445 / 2007,9974 / 2000$ e 9966/2000, RDC n ${ }^{\circ}$ 306/2004 e Resolução CONAMA n ${ }^{\circ}$ 358/2005 envolvem resíduos de saúde e são, dessa forma, contempladas pela Política Nacional de Resíduos Sólidos (PNRS), diretrizada pela Lei $\mathrm{n}^{\circ} 12.305 / 2010$ e regulamenta pelo Decreto $\mathrm{n}^{\circ} 7404 / 2010$. Trata-se de uma normativa voltada para tratar não somente da disposição e controle, mas de questões que recentemente ganharam destaque, como a logística reversa e a necessidade de plano de resíduos sólidos, a fim de melhores resultados ambientais e gerais, em linhas positivas financeiras e operacionais embasadas na sustentabilidade (VENTURA, 2012).

No entanto, pesquisa conduzida na cidade de São Paulo mostrou que $76 \%$ dos pesquisados descartavam seus medicamentos no lixo comum, $6 \%$ na pia ou no vaso sanitário e $92,5 \%$ afirmaram que nunca procuraram informações sobre descarte de fármacos (MELO, 2005). São formas de descarte selecionadas, a fim de sanar rapidamente o incômodo da posse do medicamento (ALVARENGA; NICOLETTI, 2010), mas que podem repercutir na saúde humana e ambiental (ZUCCATO et al., 2006).

O descarte de medicamentos no esgoto ou lixo comum leva os produtos às estações e locais de coleta e tratamento desses detritos e pode contaminar o ambiente e gerar riscos à saúde pública (CARVALHO et al., 2009).

No entanto, o lixo comum é o destino mais frequente dos medicamentos indesejados, em especial, pelo desconhecimento 
do usuário final de outras formas de descarte. Isso acrescenta à questão o problema da biossegurança dos indivíduos, que podem contatar esses resíduos, em geral, catadores da coleta e seleção, cuja saúde se posiciona como um dos elos frágeis da deposição em lixo comum de medicamentos. Há pouca previsão legal sobre o consumidor final no descarte de fármacos e baixa informação e opções para o recolhimento desses produtos, bem como poucos aterros sanitários próprios, incineradores habilitados (GLASSMEYER et al., 2009; ALVARENGA; NICOLETTI, 2010;) e baixo gerenciamento das farmácias e estabelecimentos de saúde sobre o destino final desses produtos (JOÃO, 2011).

É uma condição plurifatorial, em que fármacos têm sido identificados com frequência em águas superficiais, águas subterrâneas e no solo (EICKHOFF; HEINECK; SEIXAS, 2009). Em razão de seus atuais impactos e do extensivo emprego, na medicina humana e veterinária, são considerados contaminantes ambientais emergentes (FENT; WESTON; CAMINADA, 2006), cujos impactos ambientais do descarte inadequado têm sido interesse de estudo no mundo todo, na forma de atuais contaminantes do solo e da água de origem doméstica ou mesmo oriundos da saúde (ZUCCATO et al., 2006).

Nas águas, a presença mais comum é de analgésicos, antipiréticos, antilipidêmicos, anti-inflamatórios, betabloqueadores, hormônios esteroides, compostos neuroativos, antiparasitários e anti-hipertensivos, com frequência em concentrações superiores a 1 micrograma por litro. Medicamentos como Hidrocloritiazida, Ibuprofeno, Benzafibrato, Atenolol, Ofloxacino, Furosemida, Eritromicina, Claritromicina, Lincomicina e Carbamazepina são muito frequentes (FENT; WESTON; CAMINADA, 2006).

Os impactos ambientais e sobre a saúde humana de muitos desses medicamentos são desconhecidos. Outros, contudo, são de efeito conhecido: os antibióticos no ambiente geram maior resistência de bactérias, o que pode reduzir a eficácia de tratamento pela menor susceptibilidade (GHISELLI; JARDIM, 2007).

Os estrogênios podem afetar o sistema reprodutivo de organismos que vivem no meio, causando fenômenos como a feminização de peixes machos e alterações no sistema reprodutivo e neurológico, podendo ocasionar disfunções tireoidianas e cânceres de mama, próstata e testículos, além da queda na produção de espermatozoides. Os imunossupressores e antineoplásicos utilizados em quimioterapia são, por sua vez, potenciais agentes mutagênicos (MARTINEZ, 2009; MORLEY, 2009; JOÃO, 2011).

São medicamentos muito consumidos, embora suas concentrações ambientais não possam ser atribuídas somente ao uso natural e excreção, pois as estações de tratamento, nesses casos, removeriam total ou parcialmente seu impacto. No entanto, se depositados integralmente no meio ou em vias que levem a esse (como esgoto e lixo comum) atingem o ambiente em forma original, sem passar pelo metabolismo humano, o que acentua o seu potencial poluente (DAUGHTON, 2003).

O conhecimento sobre como ocorre o descarte de fármacos indesejados é relevante pelo potencial biologicamente ativo desses produtos sobre organismos terrestres e aquáticos, mesmo em condição-traço. Pouco se conhece ainda sobre os efeitos crônicos da exposição aos fármacos e se sabe ainda que a mistura de diferentes produtos pode ampliar a toxidade em novas misturas resultantes. Fatores que podem trazer resultados imprevisíveis de interação, capazes de impactos de gravidade de curto, médio e longo prazos, inclusive, em locais de nascentes (FLAHERTY; DODSON, 2005; BOUND; VOULVOULIS, 2006). São os riscos ambientais emergentes da prática (GLASSMEYER et al., 2009).

O consumidor final é uma peça fundamental na resolução do problema, mas para que assuma atitude positiva nessa cadeia, requer a formação da consciência ambiental, que é embrionária em relação aos medicamentos, e o acesso à informação, a fim de evitar a contaminação do meio e seus riscos (BUENO; WEBER; OLIVEIRA, 2009; VETTORAZZI, VENAZZI, 2008).

Uma caracterização de medicamentos descartados da forma mais frequente, o lixo comum, permite identificar, em um espaço de interesse, quais são os fármacos mais presentes, quem são os consumidores de maior atenção e quais os principais atores ativos desse descarte. No Brasil, o descarte de medicamentos em desuso, vencidos ou sobras é feito por grande parte da população em lixo comum ou em rede pública de esgoto. Esse tipo de procedimento gera agressão ao meio ambiente, a contaminação da água, do solo e de animais, além do risco à saúde de pessoas que possam reutilizá-los por acidente ou mesmo, intencionalmente, em função de fatores sociais ou circunstanciais diversos (VETTORAZZI; VENAZZI, 2008).

Com isso, investigar como é feito o descarte de medicamentos em desuso, vencidos ou sobras é um tema que contempla a linha de pesquisa: Sociedade, Ambiente e Desenvolvimento Regional Sustentável, principalmente, de municípios que não possuem aterro sanitário, comuns no interior brasileiro, como ocorre no Município de Terenos, Mato Grosso do Sul.

Para isso, foi realizada uma catalogação dos medicamentos descartados no lixo urbano pela população em um período de 12 meses, a fim de orientações mitigativas para as Unidades Básicas de Saúde e farmácias no ato do fornecimento de medicamentos, com informações pontuais ao público com a finalidade de melhoria das práticas de descarte, eliminando ou minorando, significativamente, as não conformidades encontradas e seus potenciais riscos à saúde humana e ambiental

O objetivo geral deste artigo é conhecer, observar e discutir o comportamento da população de Terenos, Mato Grosso do Sul (formada por usuários dos serviços da UBS, PSF e das drogarias) frente ao uso, aos cuidados e o descarte de medicamentos, classificação, caracterização e composição 
desses medicamentos e relacionar com os ambientais e à saúde. Trata-se da abordagem do perfil da disposição final de medicamentos inválidos ou não totalmente utilizados, desenvolvida com os objetivos acessórios de a) levantar os medicamentos distribuídos e comercializados no município; b) classificar os medicamentos quanto a sua função terapêutica e c) levantar os medicamentos em desuso e devolvidos às Unidades Básicas de Saúde (UBS), Programas de Saúde da Família (PSF) e drogarias em comparação com as quantidades identificadas no lixo doméstico.

\section{Material e Métodos}

Trata-se de pesquisa descritivo-exploratória a respeito da caracterização do descarte de medicamentos no lixo comum do município de Terenos, Mato Grosso do Sul. A coleta a campo, que serviu de análise, foi realizada, em 2015 e 2017, na Unidade de Tratamento Residual (UTR) do município de Terenos (Mato Grosso do Sul, MS, Brasil), em três etapas: contato com o gerente responsável pela UTR, para autorização da coleta e levantamento dos resíduos; visita in loco e reunião junto aos catadores, que operam no local, em que houve a orientação na coleta dos medicamentos e insumos farmacêuticos e hospitalares descartados, sobre riscos de biossegurança que podem ocorrer na separação e contato com o material, de acordo com a legislação (BRASIL, 2004, 2005) e coleta, no período de 15 dias corridos do lixo farmacêutico e hospitalar, separado em sacos brancos com capacidade de 100 litros, devidamente identificados como resíduos infectantes.

O Projeto teve aprovação do Comitê de Ética em Pesquisa com seres humanos da Universidade Anhanguera - Uniderp, sob o Número do Parecer: 2.307.289. Conforme instruções da Resolução 466/12 para pesquisas com seres humanos, todas as pessoas entrevistadas durante a execução da pesquisa assinaram um Termo de Consentimento Livre e esclarecido (TCLE).

As análises e caracterizações do descarte de medicamentos no Brasil possuem alta heterogeneidade de métodos, o que torna impossível uma análise a respeito. No entanto, torna viável a consideração dos resultados, a fim de subsidiar criticamente os achados por estruturas metodologicamente ajustadas aos campos de pesquisa.

Assim, optou-se pela caracterização física dos resíduos farmacêuticos e hospitalares encontrados na unidade considerada, método baseado dentro do qual a composição total é considerada e, após, dividida na parte ou partes de interesse (PRANDINI et al., 2000).

Os catadores identificaram o conteúdo geral deposto e selecionaram os medicamentos, que foram separados conforme os objetivos desta pesquisa. Um dos fatores que possibilitou essa caracterização e a eficiência do período de corte foi o pequeno porte do município de Terenos, campo de estudo.

Quanto ao período de corte temporal, no estudo "Gerenciamento de resíduos sólidos gerados em laboratório de análises clínicas da cidade de Ribeirão Preto - São Paulo, 2007: um estudo de caso", de autoria de Silva (2008), foi utilizado mesmo período e uso de coletas espaçadas de dias. Os resultados de seu estudo mostraram excelente apuro metodológico na caracterização dos resíduos pretendidos. Da mesma forma que na pesquisa mencionada, neste artigo os materiais foram recebidos em sacos e separados pela ação dos catadores e efetuado em mesmo período de tempo de avaliação, com a preleção feita no ambiente de geração dos resíduos.

Os medicamentos descartados foram separados e divididos em classes, conforme descrição, tipo do produto, validade e quantidade. Foram ainda verificados os dez principais medicamentos com maior descarte por meio da quantificação dos achados.

Os dados coletados foram tabulados e tratados estatisticamente pelo programa Microsoft Excel $2016^{\circledR}$ e o referencial de literatura foi criticamente analisado e transcrito, a fim de dar suporte para discussão dos achados, junto da legislação vigente (BRASIL, 2004, 2005).

\section{Resultados e Discussão}

A população estimada da cidade de Terenos, para o ano de 2016, foi de 19.914 habitantes, 7.275 desses na área urbana (IBGE, 2010). Com a coleta dos medicamentos no lixo comum, na Unidade de Tratamento de Resíduos (UTR) municipal de Terenos, forneceu 476 amostras de medicamentos e/ ou insumos farmacêuticos. As amostras foram separadas e classificadas.

$\mathrm{Na}$ amostragem classificada, $0,4 \% \quad(2,2$ g) eram fitoterápicos, 4,8\% (25 g) eram produtos oficinais manipulados e $93 \%(n=89,6 \mathrm{~g})$ especialidades farmacêuticas. No todo dos medicamentos registrados, 50,8\% $(n=242)$ estavam dentro do prazo de validade, $40,5 \%(n=193)$ vencidas e $8,6 \%(n=41)$ sem identificação de validade.

O Quadro 1 apresenta a quantificação dos medicamentos coletados, que foi dividida em trinta grupos, com especificação de sua classe terapêutica, quantidade de amostras com e sem conteúdo e respectivo percentual no todo descartado. Os 476 descartes lançaram no lixo comum da UTR o montante de $507,6 \mathrm{~g}$ de medicamentos e/ou insumos farmacêuticos em um período de 15 dias. Em projeção, mantendo o padrão de descarte, a recepção anual da UTR seria de cerca de $12,1 \mathrm{~kg}$ de fármacos, em uma média de descarte anual em lixo comum de $1,67 \mathrm{~g}$ por habitante. 
Quadro 1 - Quantificação e classificação dos medicamentos e/ou insumos farmacêuticos coletados em UTR 2015 e 2017, em Terenos, Mato Grosso do Sul, Brasil, 2017

\begin{tabular}{|c|c|c|c|c|}
\hline Classe Terapêutica & \begin{tabular}{|l|} 
Amostras com \\
Medicamento
\end{tabular} & $\begin{array}{c}\text { Amostras } \\
\text { Vazias }\end{array}$ & $\begin{array}{c}\text { Total de } \\
\text { Amostras }\end{array}$ & $\%$ \\
\hline Anti-inflamatório e relaxantes musculares & 75 & 5 & 80 & 16,8 \\
\hline Anti-hipertensivos & 61 & 11 & 72 & 15,1 \\
\hline Antibióticos e antibacterianos & 56 & 6 & 62 & 13,0 \\
\hline Diuréticos & 24 & 6 & 30 & 6,3 \\
\hline Antiulcerosos & 20 & 9 & 29 & 6,1 \\
\hline Antilipêmicos & 20 & 6 & 26 & 5,5 \\
\hline Amostras sem identificação & 24 & 0 & 24 & 5,0 \\
\hline Hipoglicemiantes & 23 & 0 & 23 & 4,8 \\
\hline Antidepressivos & 16 & 6 & 22 & 4,6 \\
\hline Digestivos e antieméticos & 15 & 1 & 16 & 3,4 \\
\hline Suplementos, vitaminas e sais minerais & 13 & 1 & 14 & 2,9 \\
\hline Antialérgicos & 11 & 1 & 12 & 2,5 \\
\hline Antivertiginosos e vasodilatadores & 10 & 1 & 11 & 2,3 \\
\hline Analgésicos & 8 & 0 & 8 & 1,7 \\
\hline Antigases & 7 & 0 & 7 & 1,5 \\
\hline Reidratantes orais & 6 & 0 & 6 & 1,3 \\
\hline Vermífugos & 5 & 0 & 5 & 1,1 \\
\hline Analgésicos e antiespasmódicos & 5 & 0 & 5 & 1,1 \\
\hline Expectorantes e bronco dilatadores & 4 & 0 & 4 & 0,8 \\
\hline Fitoterápicos & 3 & 0 & 3 & 0,6 \\
\hline Homeopáticos & 3 & 0 & 3 & 0,6 \\
\hline Antifúngico & 2 & 0 & 2 & 0,4 \\
\hline Antiarrítmicos & 2 & 0 & 2 & 0,4 \\
\hline Hormônios & 2 & 0 & 2 & 0,4 \\
\hline Antivirais & 2 & 0 & 2 & 0,4 \\
\hline Antigripais & 2 & 0 & 2 & 0,4 \\
\hline Glicosídeo cardiotónico & 1 & 0 & 1 & 0,2 \\
\hline Laxantes & 1 & 0 & 1 & 0,2 \\
\hline Ansiolítico & 1 & 0 & 1 & 0,2 \\
\hline Antipsicótico & 1 & 0 & 1 & 0,2 \\
\hline Antilipêmicos & 20 & 6 & 26 & 5,5 \\
\hline
\end{tabular}

Fonte: Dados da pesquisa.

A quantidade da estimativa pode ser superior em razão da não quantificação em mg ou g de insumos como soluções de manipulação, cuja composição não pode ser especificada. A prática de descarte de medicamentos no lixo comum é amplamente utilizada para a destinação de embalagens com conteúdo ( $\mathrm{n}=423$ embalagens - 88,8\%), válidas ou não. As embalagens vazias correspondem a um pequeno montante, não expressivo ( $\mathrm{n}=53$ embalagens - 11,1\%).

Dez medicamentos apresentaram maior volume $(\mathrm{mg} / \mathrm{g})$ de descarte: foram esses o Ibuprofeno $(n=83,1 \mathrm{~g}-16,9 \%)$, a Metformina $(n=68,8 \mathrm{~g}-14 \%)$, a Amoxicilina $(n=55,75 \mathrm{~g}-$ $11,4 \%)$, a Cefalexina $(n=43 \mathrm{~g}-8,8 \%)$, o Ciprofloxacino $(\mathrm{n}=$ $35 \mathrm{~g}-7,1 \%$ ), a Nimesulida ( $\mathrm{n}=26,4 \mathrm{~g}-5,4 \%$ ), o Cloreto de Sódio ( $\mathrm{n}=21 \mathrm{~g}-4,3 \%)$, o Sulfametoxazol $(\mathrm{n}=15,6 \mathrm{~g}-3,2 \%)$, a Ranitidina ( $\mathrm{n}=11,85 \mathrm{~g}-2,4 \%)$ e o Propranolol $(\mathrm{n}=10,32 \mathrm{~g}$ $-2,1 \%$ ). As medidas em $\mathrm{g}$ dos descartes são apresentadas na sequência, bem como listagem dos produtos e montante de descarte.

Em relação às classes de medicamentos mais frequentemente descartados, o grupo de três principais produtos identificados contém anti-inflamatórios e relaxantes musculares, anti-hipertensivos e antibióticos e antibacterianos, correspondendo respectivamente a $20,8 \%(\mathrm{n}=80), 18,8 \%(\mathrm{n}=$ $72)$ e $16,1 \%(n=62)$ do total de produtos descartados.

A UTR do município de Terenos recebe o lixo gerado na zona urbana do município, o que levou ao fechamento de sua população aos aproximados 7.275 habitantes (IBGE, 2010). As medicações foram prevalentes em especialidades farmacêuticas, com relativa paridade no volume de descartes entre medicamentos vencidos e dentro do prazo de validade, respectivamente $40,5 \%(n=193)$ e $50,8 \%(n=242)$.

É um resultado que mostra um perfil diferenciado no uso e guarda de medicamentos se comparado com o que a literatura menciona. Análise do descarte de medicamentos na cidade de Caçado, Santa Catarina (Rio Grande do Sul, RS), indicou que entre os fármacos indesejáveis descartados, $90 \%$ eram vencidos e $10 \%$ dentro do prazo de validade (JUNIO; ZANCANARO, 2013).

Pode colaborar para essa situação diferenciada o interesse do descarte de medicamentos sobressalentes ou sem uso 
corrente. Cerca de $37 \%$ dos usuários de medicamentos, em pesquisa empregada no município de Ijuí (Rio Grande do Sul, RS), têm o hábito de preservar sobras de medicamento após o fim do tratamento. São produtos que têm maiores chances de armazenagem prolongada e/ou inadequada, acompanhada de riscos de efeitos adversos do uso, em possível automedicação. As mesmas sobras de tratamento podem ser descartadas antes de seu vencimento, a fim de terminar com eventuais inconvenientes da posse do produto (BUENO; WEBER; OLIVEIRA, 2009).

A presença de sobras também é relacionada com eventuais erros de prescrição ou de dispensação dos medicamentos, bem como da apresentação do fármaco de modo a resultar em uma quantidade superior à necessária ao tratamento. Como em muitos casos não é possível fracionar os produtos, as sobras muitas vezes são inevitáveis (EICKHOFF; HEINECK; SEIXAS, 2009).

Em posse do produto, o consumidor final termina como um elo frágil na cadeia de informações a respeito do seu descarte e encaminhamento. Embora autores afirmem a respeito de boas políticas de informação e incentivo da população, a fim da entrega de medicamentos vencidos ou não em farmácias ou unidades de saúde (PROENÇA, 2011), os sinalizadores de descarte obtidos neste estudo indicam para a ineficiência dessas políticas de orientação na população avaliada.

Pelas dificuldades de prescrição, pela automedicação ou fatores ligados ao consumidor final, estima-se que $20 \%$ dos medicamentos sejam descartados no lixo comum ou rede de esgoto (ALVARENGA; NICOLETTI, 2010).

Neste estudo, 88,8\% ( $n=423)$ das embalagens foram descartadas com conteúdo, o que aponta para a necessidade de investigação a respeito da cultura de atendimento médico, prescrição e dispensação da população estudada, a fim de identificar os fatores de abundância nas sobras de fármacos.

A elevada presença de embalagens descartadas com medicamentos e/ou insumos farmacêuticas, inclusive, dentro da validade ou mesmo intactas, foi identificada de modo similar em levantamento realizado na cidade de Caçador, Rio Grande do Sul. No estudo, a maior parte dos medicamentos descartados apresentava pouco ou nenhum uso, indicativo do desperdício de verbas em sua aquisição, quer sejam públicas ou privadas (TESSARO; ZANCANARO, 2013).

É uma tendência de desperdício, que é verificada em países subdesenvolvidos e em desenvolvimento, que amplia os gastos em saúde e que compromete a possibilidade de maiores e melhores investimentos no setor, a fim de subsidiar os desperdícios (AQUINO, 2008).

A população do município estudado, com 7.275 habitantes, apresentou uma média projetada de descarte de medicamentos anual de 1,67 g por munícipe. Em avaliação de um semestre do descarte de medicamentos domiciliares, em pontos de coleta no município de Caçador, Santa Catarina (Rio Grande do Sul, RS), estudo registrou média estimada mensal de cerca de cinco quilos de descarte de fármacos para o ano de 2013, o que gerou uma faixa média anual de $0,36 \mathrm{~g}$ de fármaco por habitante (TRIBESS JUNIOR; ZANCANARO, 2013).

As quantificações apresentadas seguiram a quantidade de descarte (número de embalagens) por grupo de integração e gramas descartadas (g), por medicamento, a fim de melhor identificação dos produtos e classes terapêuticas. Na sequência (Quadro 2) é apresentada a quantificação do principal grupo de descarte, anti-inflamatórios e relaxantes musculares.

Quadro 2 - Tipo, apresentação, laboratório e quantificação dos anti-inflamatórios encontrados em UTR municipal entre 2015 e 2017. Terenos, Mato Grosso do Sul, Brasil, 2017

\begin{tabular}{|c|c|c|c|c|}
\hline \multicolumn{5}{|c|}{ Comprimido } \\
\hline Descrição & $\mathrm{mg}$ & Laboratório & Quantidade (und) & Total mg/g \\
\hline \multirow[t]{2}{*}{ Ciclobenzaprina } & 5 & Neo Química & 7 & 35 \\
\hline & & Ems & 19 & 95 \\
\hline \multicolumn{5}{|c|}{ Comprimido } \\
\hline Descrição & $\mathrm{mg}$ & Laboratório & Quantidade (und) & Total mg/g \\
\hline Subtotal mg & 26 & 130 & Subtotal mg & 26 \\
\hline Subtotal g & & & & 0,1 \\
\hline Diclofenaco & 50 & Multilab & 20 & 1.000 \\
\hline \multicolumn{3}{|c|}{ Subtotal mg } & 20 & 1.000 \\
\hline \multicolumn{3}{|c|}{ Subtotal g } & & 1 \\
\hline \multirow[t]{7}{*}{ Ibuprofeno } & 300 & Neo Química & 60 & 18.000 \\
\hline & & Multilab & 97 & 29.100 \\
\hline & & Ems & 8 & 2.400 \\
\hline & & Biosintética & 6 & 1.800 \\
\hline & 600 & Ems & 34 & 20.400 \\
\hline & & Neo Química & 10 & 6.000 \\
\hline & & Eurofarma & 9 & 5.400 \\
\hline \multicolumn{3}{|c|}{ Subtotal mg } & 224 & 83.100 \\
\hline \multicolumn{3}{|c|}{ Subtotal g } & & 83,1 \\
\hline
\end{tabular}




\begin{tabular}{|c|c|c|c|c|}
\hline Meloxicam & 7,5 & Eurofarma & 3 & 22,5 \\
\hline & 15 & Biosintética & 12 & 180 \\
\hline \multicolumn{3}{|c|}{ Subtotal mg } & 15 & 202,5 \\
\hline \multicolumn{3}{|c|}{ Subtotal g } & & 0,2 \\
\hline \multirow[t]{3}{*}{ Nimesulida } & 100 & Vitapam & 224 & 22.400 \\
\hline & & Neo Química & 35 & 3.500 \\
\hline & & Eurofarma & 5 & 500 \\
\hline \multicolumn{3}{|c|}{ Subtotal mg } & 264 & 26.400 \\
\hline \multicolumn{3}{|c|}{ Subtotal g } & & 26,4 \\
\hline \multicolumn{4}{|r|}{ Total geral mg } & $110.832,5$ \\
\hline & & & Total geral g & 110,8 \\
\hline
\end{tabular}

Fonte: Dados da pesquisa.

A segunda categoria de medicamentos descartados é o contínuo, que integram tratamentos de longo prazo (Quadro grupo de hipertensivos, medicamentos em maioria de uso

Quadro 3 - Tipo, apresentação, laboratório e quantificação dos anti-hipertensivos encontrados em UTR municipal entre 2015 - 2017. Terenos, Mato Grosso do Sul, Brasil, 2017

\begin{tabular}{|c|c|c|c|c|}
\hline \multicolumn{5}{|c|}{ Comprimido } \\
\hline Descrição & $\mathrm{mg}$ & Laboratório & Quantidade (und) & Total mg/g \\
\hline Anlodipino & 5 & Geolab & 10 & 50 \\
\hline & 10 & Vitapam & 10 & 100 \\
\hline & & Geolab & 120 & 1.200 \\
\hline \multicolumn{3}{|c|}{ Subtotal mg } & 140 & 1.350 \\
\hline \multicolumn{3}{|c|}{ Subtotal g } & & 1,35 \\
\hline Atenolol & 50 & Vitapam & 27 & 1.350 \\
\hline & & Prati & 4 & 200 \\
\hline & & Furp & 1 & 50 \\
\hline \multicolumn{3}{|c|}{ Subtotal mg } & 32 & 1.600 \\
\hline \multicolumn{3}{|c|}{ Subtotal $g$} & & 1,6 \\
\hline Captopril & 25 & Furp & 12 & 300 \\
\hline \multicolumn{3}{|c|}{ Subtotal mg } & 12 & 300 \\
\hline \multicolumn{3}{|c|}{ Subtotal $g$} & & 0,3 \\
\hline \multirow[t]{4}{*}{ Enalapril } & 5 & Roytom & 20 & 100 \\
\hline & 10 & Cimed & 49 & 490 \\
\hline & 20 & Cimed & 40 & 800 \\
\hline & & Teuto & 108 & 2.160 \\
\hline \multicolumn{3}{|c|}{ Subtotal mg } & 217 & 3.550 \\
\hline \multicolumn{3}{|c|}{ Subtotal g } & & 3,5 \\
\hline \multirow[t]{2}{*}{ Losartana } & 50 & Prati & 48 & 2.400 \\
\hline & & Geolab & 9 & 450 \\
\hline \multicolumn{3}{|c|}{ Subtotal mg } & 57 & 2.850 \\
\hline \multicolumn{3}{|c|}{ Subtotal g } & & 2,85 \\
\hline Metildopa & 250 & Tks & 10 & 2.500 \\
\hline \multicolumn{3}{|c|}{ Subtotal mg } & 10 & 2.500 \\
\hline \multicolumn{3}{|c|}{ Subtotal g } & & 2,5 \\
\hline \multirow[t]{2}{*}{ Propranolol } & 40 & Osório & 227 & 9.080 \\
\hline & & Furp & 31 & 1.240 \\
\hline \multicolumn{3}{|c|}{ Subtotal mg } & 258 & 10.320 \\
\hline \multicolumn{3}{|c|}{ Subtotal g } & & 10,3 \\
\hline \multicolumn{3}{|c|}{ Total geral mg } & 726 & 22.470 \\
\hline \multicolumn{3}{|c|}{ Total geral g } & & 22,5 \\
\hline
\end{tabular}

Fonte: Dados da pesquisa.

Aterceira classe mais comum identificada no descarte foram os antibióticos e antibacterianos, medicamentos utilizados, muitas vezes, em prescrições nos pronto-atendimentos e boa parte dependente de receita médica (Quadro 4). 
Quadro 4 - Tipo, apresentação, laboratório e quantificação dos antibióticos e antibacterianos encontrados em UTR municipal entre 2015 - 2017, Terenos, Mato Grosso do Sul, Brasil, 2017

\begin{tabular}{|c|c|c|c|c|}
\hline \multicolumn{5}{|c|}{ Cápsula } \\
\hline Descrição & $\mathrm{Mg}$ & Laboratório & Quantidade (und) & Total mg/g \\
\hline \multirow[t]{4}{*}{ Amoxicilina } & 500 & Furp & 25 & 12.500 \\
\hline & & Prati & 14 & 7.000 \\
\hline & & Multilab & 4 & 2.000 \\
\hline & & Agila & 21 & 10.500 \\
\hline Subtotal mg & & & & 32.000 \\
\hline Subtotal g & & & & 32,0 \\
\hline \multicolumn{5}{|c|}{ Comprimidos } \\
\hline Descrição & $\mathrm{Mg}$ & Laboratório & Quantidade (und) & Total mg/g \\
\hline Azitromicina & 500 & Prati & 1 & 500 \\
\hline \multicolumn{2}{|l|}{ Subtotal mg } & & & 500 \\
\hline \multicolumn{2}{|l|}{ Subtotal g } & & & 0,5 \\
\hline \multirow{2}{*}{ Cefalexina } & \multirow{2}{*}{500} & Furp & 57 & 28.500 \\
\hline & & Teuto & 29 & 14.500 \\
\hline Subtotal mg & & & & \\
\hline Subtotal g & & & & \\
\hline & & Ems & 56 & 28.000 \\
\hline Ciprofloxacino & 500 & Prati & 4 & 2.000 \\
\hline & & Labóris & 10 & 5.000 \\
\hline Subtotal mg & & & & \\
\hline Subtotal g & & & & \\
\hline Metronidazol & 250 & Furp & 9 & 2.250 \\
\hline Subtotal mg & & & 6 & 2.250 \\
\hline Subtotal g & & & & 2,2 \\
\hline & & Furp & 19 & 1.520 \\
\hline $\begin{array}{l}\text { SulTametoxazol + } \\
\text { trimetoprima }\end{array}$ & $400+80$ & Teuto & 10 & 4000 \\
\hline & & Biofarma & 10 & 4000 \\
\hline Subtotal sulfametor & zol mg & & & 15.600 \\
\hline Subtotal sulfametor & $\mathrm{zol} \mathrm{g}$ & & & 15,6 \\
\hline Subotal trimetoprin & $\mathrm{mg}$ & & & 3.120 \\
\hline Subtotal trimetropr & na $g$ & & & 3,1 \\
\hline Amoxicilina & $250 / 5$ & Prati & 475 & 23.750 \\
\hline Subtotal mg & & & 475 & 23.750 \\
\hline Subtotal g & & & & 23,7 \\
\hline & & & midos & \\
\hline Ampicilina & $250 / 5$ & Neo química & 15 & 750 \\
\hline Subtotal mg & & & 15 & 750 \\
\hline Subtotal g & & & & 0,7 \\
\hline Cefalexina & $250 / 5$ & Furp & 175 & 8.750 \\
\hline Subtotal mg & & & 175 & 8.750 \\
\hline Subtotal g & & & & 8,7 \\
\hline & & & & \\
\hline Descrição & $\mathrm{mg} / \mathrm{g}+\mathrm{UI} / \mathrm{g}$ & Laboratório & Quantidade (und) & Total mg/g \\
\hline Sulfato de & & \begin{tabular}{|l|} 
Nycomed \\
\end{tabular} & 3,8 & 19,0 \\
\hline $\begin{array}{l}\text { neomicina }+ \\
\text { bacitracina }\end{array}$ & $5+250$ & Neo Química & 3,7 & 18,5 \\
\hline Subtotal sulf. de ne & micina $\mathrm{mg}$ & & 7,5 & 37,5 \\
\hline Subtotal sulf. de ne & micina $\mathrm{g}$ & & & 0,3 \\
\hline Subtotal bacitracin & $\mathrm{UI} / \mathrm{g}$ & & & 1.875 \\
\hline Subtotal bacitracin & & & & 12,5 \\
\hline Subtotal bacitracin & & & & 0,1 \\
\hline & & & & \\
\hline Descrição & $\mathrm{mg} / \mathrm{mL}$ & Laboratório & Quantidade (und) & Total $\mathrm{mg} / \mathrm{g}$ \\
\hline Rifamicina Sódica & 20 & Eurofarma & 15 & 300 \\
\hline Subtotal mg & & & 15 & 300 \\
\hline Subtotal g & & & & 0,3 \\
\hline Total mg & & & & 165.070 \\
\hline Total g & & & & 165,0 \\
\hline
\end{tabular}

Fonte: Dados da pesquisa. 
Um grupo de medicamentos chama a atenção em relação ao desconhecimento e gravidade de seu descarte no ambiente. São hormônios, sobre os quais o foco da ecotoxidade está sendo amplamente investigado (Quadro 5).

Quadro 5 - Tipo, apresentação, laboratório e quantificação dos hormônios encontrados em UTR municipal entre 2015-2017. Terenos, Mato Grosso do Sul, Brasil, 2017

\begin{tabular}{|c|c|c|c|c|}
\hline \multicolumn{5}{|c|}{ Comprimido } \\
\hline Descrição & $\mathrm{mg}$ & Laboratório & $\begin{array}{l}\text { Quantidade } \\
\text { (und) }\end{array}$ & $\begin{array}{l}\text { Total } \\
\mathrm{mg} / \mathrm{g}\end{array}$ \\
\hline Levotiroxina & 100 & Merck & 4 & 400 \\
\hline \multicolumn{3}{|l|}{ Subtotal mg } & & 400 \\
\hline \multicolumn{3}{|l|}{ Subtotal g } & & 0,4 \\
\hline Levonorgestrel & 0,15 & $\begin{array}{c}\text { União } \\
\text { Química }\end{array}$ & 18 & 2,7 \\
\hline \multicolumn{5}{|c|}{ Comprimido } \\
\hline \multicolumn{3}{|l|}{ Subtotal mg } & & 2,7 \\
\hline \multicolumn{3}{|l|}{ Subtotal $g$} & & 0,02 \\
\hline Etinilestradiol & 0,03 & $\begin{array}{c}\text { União } \\
\text { Química }\end{array}$ & 18 & 0,54 \\
\hline \multicolumn{3}{|l|}{ Subtotal mg } & & 0,54 \\
\hline \multicolumn{3}{|l|}{ Subtotal g } & & $<0,01$ \\
\hline \multicolumn{4}{|l|}{ Total geral mg } & 403,2 \\
\hline \multicolumn{4}{|l|}{ Total geral g } & 0,4 \\
\hline
\end{tabular}

Fonte: Dados da pesquisa.

Os demais medicamentos e/ou insumos farmacêuticos encontrados em descarte são quantificados na sequência, em quadros que apresenta a sua descrição (princípio ativo), os miligramas da apresentação da embalagem original, laboratório, unidades/gramas/miligramas ou unidade de medida referencial identificada e total em $\mathrm{mg}$ apresentado, quantificando a totalidade dos produtos coletados durante os 15 dias de investigação da UTR de Terenos, Mato Grosso do Sul.

Foram coletadas ainda 131 cápsulas de cartelas sem identificação do laboratório Pharmamed e 11 sachês igualmente não identificados do Laboratório São Bento, não quantificados em razão da impossibilidade de identificação do seu princípio ativo.

Considerando as diferenças metodológicas da coleta efetuada, que ocorreu em pontos estratégicos de postos de saúde e farmácia-escola do município pesquisado e o estímulo ao descarte que foi feito à população, a faixa mensal obtida se mostrou muito inferior a encontrada neste estudo, ressaltando a possível emergência do problema ambiental e de saúde do descarte em lixo comum de medicamentos.

Sabe-se que os equívocos de descarte de medicação representam uma das três principais causas de intoxicação por fármacos no Brasil (MELO, 2005). No entanto, a preferência pelo descarte em lixo comum é prevalente: em pesquisa na população do Estado de São Paulo, com 141 participantes, foi constatada a predileção pelo lixo comum para o descarte de fármacos indesejáveis entre $89 \%$ dos consumidores de medicamentos (MAROSTEGA; PAVAN, 2009; UEDA; TAVERNARO, 2009).
Resultados semelhantes foram obtidos no Kuwait, por Abahussain; Ball (2007), o questionário aplicado a 200 indivíduos sobre como descartavam os medicamentos, $97 \%$ dos pesquisados responderam que o destino final era lixo doméstico.

Entre acadêmicos de duas Instituições de Ensino Superior (uma pública e uma privada) da cidade de Paulínia, São Paulo/Brasil, em outubro de 2011, foram obtidos resultados similares: $91 \%$ dos pesquisados afirmaram descartar seus medicamentos no lixo comum ou em água corrente. Os demais relataram descartar em postos de saúde, farmácias, drogarias e centros comunitários (PROENÇA, 2011).

Em pesquisa na cidade de São Paulo, foi observada a adoção do lixo comum para o descarte de medicamentos por $76 \%$ dos pesquisados como destino final para seus fármacos (MELO, 2005) e é baixo o nível de preocupação e consciência ambiental a respeito dos efeitos desse descarte.

Em pesquisa entre residentes da cidade de São Paulo, a Fundação Oswaldo Cruz identificou que o lixo comum foi o destino escolhido por $75,3 \%$ dos entrevistados em sua avaliação. Entre os investigados, 9,8\% afirmaram nunca descartar medicamentos, mesmo vencidos. A desinformação sobre como proceder a esse descarte também foi destacada, uma vez que $92,5 \%$ dos entrevistados afirmaram nunca terem recebido - tampouco solicitado - informações a respeito de como realizar esse processo (MELO, 2005).

A deposição de medicamentos em lixo comum coloca em risco o ambiente e a saúde coletiva pela deposição de produtos com pouca ou nenhuma biodegradabilidade, capazes de ter efeitos biológicos extensíveis às várias formas de vida. A maioria dos medicamentos tem o potencial de permanecer por longo tempo no ambiente e se bioacumular no solo e na água. Igualmente, podem perpassar membranas celulares e resistir à degradação química e metabólica de modo mais significativo que poluentes em geral (CARVALHO et al., 2009).

Os fármacos descartados, válidos ou não, em lixo comum chegam ao ambiente com potencial bioquímico total ou próximo desse (PROENÇA, 2011). O município investigado não possui um aterro próprio para a deposição de lixo e o mesmo ou é destinado a capital, Campo Grande (Mato Grosso do Sul, Brasil) ou depositado em espaços de reserva, a fim de posterior coleta, após passagem pela UTR.

Os fármacos se engendram nesse processo e terminam pulverizados por estes dois destinos. A ausência de uma estrutura suficiente para o recolhimento dos produtos e, também, para o seu processamento após o descarte são fatores que elevam as práticas inadequadas de descarte (ALVARENGA; NICOLETTI, 2010).

Quando aterrados, em curto prazo, pode haver redução dos efeitos deletérios, mas chuvas e umidade podem formar um lixiviado de moléculas dos materiais impregnados no aterro, gerando um produto acidental igualmente capaz de impactos sobre o ambiente. Inconvenientes em longo prazo tão ou mais graves quanto à deposição não aterrada (GLASSMEYER et 
al., 2009).

Da deposição como é feita, surge a possível situação a respeito dos impactos ambientais e de saúde da presença de fármacos no ambiente: o destino do solo, que recebe essa deposição, se torna um problema emergente às pessoas e ao meio. Caso mais tarde seja utilizado na agricultura, por exemplo, existe a possibilidade de contaminação das culturas provenientes e há poucos estudos e práticas destinados para recuperação e descontaminação de solos por fármacos (SANTOS et al., 2010).

Dez medicamentos se destacaram na coleta como de maior expressão de descarte: o Ibuprofeno, a Metformina, a Amoxicilina, a Cefalexina, o Ciprofloxacino, a Nimesulida, o Cloreto de Sódio, o Sulfametaxazol, a Ranitidina e o Propranolol. São três grupos protagonistas de descarte: a) antiinflamatórios e relaxantes musculares; b) anti-hipertensivos e c) antibióticos e antibacterianos.

Os produtos lançados ao meio na cidade-alvo deste estudo não diferem da cartela de achados que se manifestam comuns nas águas, principal meio estudado sobre a deposição de medicamentos. São esses principalmente analgésicos, antilipêmicos, anti-inflamatórios, anti-hipertensivos e antiparasitários, substâncias como o Ibuprofeno, o Atenolol, o Ofloxacino, a Furosemida, a Carbamazepina e o Benzofibrato, além de hormônios e antidepressivos capazes de efeitos mutagênicos (FENT; WESTON; CAMINADA, 2006; GHISELLI; JARDIM, 2007; JOÃO, 2011; MARTINEZ, 2009; MORLEY, 2009).

A amostra de descarte em lixo comum, na cidade analisada, sintetiza as recorrentes amostras de água e posiciona $\mathrm{o}$ problema ambiental e de saúde coletiva consolidado na baixa informação e equívoco no descarte medicamentoso.

São achados parcialmente coerentes com os reportados em estudos como o desenvolvido na cidade de Santa Catarina, Rio Grande do Sul (Brasil), em que durante um semestre de análise em município do interior foram descartados, principalmente, hipertensivos, antibióticos e analgésicos (TRIBESS JUNIOR; ZANCANARO, 2013).

Os medicamentos hipertensivos dentro das classes mais comuns de medicamentos descartados também foi um resultado encontrado em revisão, na qual se reafirmou que essa maior deposição decorre do elevado número de casos de doenças que atingem o sistema cardiovascular, entre as quais a hipertensão arterial. Com isso, o consumo de medicamentos anti-hipertensivos é elevado, da mesma forma que o hábito da população atingida em não dar continuidade ao tratamento. Isso torna a perda desses fármacos um evento de alta expectativa (ALMEIDA; PAZ; SILVA, 2011).

Em outro estudo sobre adesão de tratamento, igualmente foi relatado o frequente hábito da descontinuidade de tratamento por parte de hipertensos: cerca de $50 \%$ dos indivíduos abandonam por completo o tratamento ou o fazem de modo parcial e descontinuado (SANTOS et al., 2005).

Os resultados gerais da coleta mostraram a presença de trinta categorias de medicamentos descartados pela população terenense, em um leque de usos variado. Entre esses estão, com alto volume de descarte, fármacos como desreguladores do sistema endócrino (hormônios), antidepressivos e antihelmínticos, medicamentos que possuem ecotoxidade relativa (SANTOS et al., 2005).

Sobre o Ibuprofeno ser o fármaco de maior deposição, é uma constatação que segue a popularidade do medicamento não apenas no Brasil e, além da comercialização, esse tem distribuição gratuitamente pelas políticas públicas de saúde instituídas no Brasil e, com isso, se torna um dos mais presentes em águas e solos. No ambiente tem o inconveniente de uma degradação bastante lenta (ALI et al., 2009).

A ecotoxidade de fármacos, como: o Ibuprofeno, o Paracetamol, a Amoxicilina, a Furosemida, o Atenolol, a Digoxina e a Anlodipino foram avaliadas com o uso de uma hidra de água doce (Hydra vulgaris), exposta por sete dias aos produtos. Não houve registro de letalidade, no entanto, não foi possível ao estudo afirmar sobre a segurança desses medicamentos no ambiente, pela quase sempre ação conjunta a outros em deposição. Outra razão é a própria variação de resposta de organismos terrestres e aquáticos que podem ser mais ou menos suscetíveis a ação dos produtos (PASCOE; KARNTANUT; MÜLLER, 2003).

Fármacos como Carbamazepina, Ibuprofeno, Diclofenaco, Captopril, Metformina, Metropolol e Propranolol foram testados a fim de verificar a sua toxidade no ambiente. Cada organismo utilizado para teste respondeu de um modo muito próprio aos fármacos, o que conduziu o pesquisador a orientar uma classificação moderada de risco quanto a esses produtos (CLEUVERS, 2003).

O principal achado, que se pode extrair do estudo dessas respostas, a respeito do objetivo de atenção ambiental e de saúde coletiva, a qual é direcionado este estudo, tem a ver com a consideração geral dos fármacos depostos no meio. Poucos produtos possuem uma toxidade elevada, como é o caso do Propranolol, que causou imobilização em cerca de $50 \%$ dos organismos animais expostos em concentração de $7,5 \mathrm{mg} \mathrm{L}^{-1}$, e a maioria costuma ter isoladamente uma baixa toxidade. No entanto, é da combinação dos diversos fármacos presentes no meio que podem surgir os principais riscos. Dois produtos de baixa toxidade juntos podem ter uma associação devastadora ao ambiente e quando e como esses encontros poderão acontecer é imprevisível havendo uma deposição descontrolada (PASCOE; KARNTANUT; MÜLLER, 2003).

Por essa razão, ainda que muitos fármacos da cartela de achados na coleta investigativa deste estudo possam se apresentar em pequenas quantidades ou mesmo ter comportamento registrado de baixa toxidade, a pluralidade das classificações terapêuticas e do número de medicamentos oferece uma possibilidade quase infinita de combinações, cujo impacto é imprevisível ao ambiente, e cujo volume de descarte é um problema que necessita ser abordado.

Ainda sobre o Ibuprofeno e, também, o ácido 
acetilsalicílico, ambos os produtos encontrados nos descartes coletados na UTR avaliada, uma avaliação realizada no ano de 2003 considerou que, entre algumas espécies de organismos, a exposição a essas substâncias ocasionaria a redução do comportamento predatório, reduzindo a quantidade e demanda alimentar. Mesmo efeito foi notado pelos autores em medicamentos igualmente presentes nas amostras consideradas, como a Amoxicilina, o Paracetamol, a Digoxina, a Furosemida, o Atenolol, a Bendroflumetiazida e o Diazepam, por exemplo (PASCOE; KARNTANUT; MÜLLER, 2003).

Existem inúmeros estudos que procuram conhecer os efeitos dos fármacos no ambiente, porém a maioria se destina às águas e aos organismos aquáticos. Pouco se sabe a respeito do solo que é o destino da maioria das deposições domésticas de medicamento.

Há sinalizações importantes que mostram os efeitos do Ciprofloxacino no ambiente. Concentrações superiores a $100 \mu \mathrm{g}$ $\mathrm{L}^{-1}$ de enrofloxina foram absorvidas por várias espécies de vegetais e convertidas no metabólito Ciprofloxacino. Sinalizadores que indicam para a importância do cuidado a respeito da disposição dos fármacos em geral no ambiente, inclusive no solo, a fim de conhecer e zelar da ecotoxidade possível e dos efeitos de bioacumulação (MIGLIORE; COZZOLINO; FIORI, 2003).

Já os estrogênicos são fármacos cuja preocupação é emergente sobre a sua presença no ambiente. São comuns nos contraceptivos orais e, em geral, seu efeito mais frequente são alterações hormonais nos organismos aos quais atingem. É o caso do Estradiol, do Etinilestradiol e da Hidroxiestrona, por exemplo, hormônios gerais cuja presença deve ser monitorada com constante atenção, a fim de regular eventuais alterações e impactos (TERNES et al., 1999).

Um estudo pioneiro a respeito dos efeitos dos estrógenos no ambiente aquático, como ocorre com a maioria dos estudos dessa natureza, permitiu a analogia geral de que os principais efeitos desses produtos são alterações endocrinológicas e reprodutivas. Um dos resultados clássicos relatados pelo estudo foi a feminilização de peixes machos, observada em efluentes no mundo todo, que contam com a presença de estrógenos (ANKLEY et al., 2007).

Entre os antidepressivos identificados nas amostras coletadas há uma quantidade prevalente de Fluoxetina. Sobre esse fármaco no ambiente, há registro de potencial ação de alterações na fertilidade (para mais) nos organismos terrestres e aquáticos do meio, podendo elevar as concentrações de estradiol entre fêmeas. Apesar do favorecimento reprodutivo, os produtos da concepção dos animais experimentados expostos ao produto apresentaram diversas anomalias ósseas e de desenvolvimento, em especial, após quatro semanas de exposição continuada. Concentrações entre 234 a $705 \mathrm{mg} \mathrm{L}^{-1}$ foram letais para $50 \%$ dos organismos expostos (crustáceo Ceriodaphnia dunia, Daphnia magna e peixe Pimephales promelas). O antidepressivo, quando associado a outros produtos depositados no ambiente, tem ainda o potencial de ampliar a sua toxidade (BROOKS et al., 2003).

Os antidepressivos, em geral, são elementos de atenção para os aspectos ambientais em razão de seu potencial de afetar efeitos neurotransmissores, mas não é possível afirmar que sua ação deletéria se encerre neste aspecto e outros danos severos podem ser de expectativa (COSTA; PLETSCH; TORRES, 2014).

Outro fator preocupante para além dos potenciais danos ao ambiente são os riscos que os medicamentos depositados em lixo comum podem oferecer a catadores, pessoas carentes e crianças, que possam vir a ter contato com os mesmos (SERAFIM et al., 2007).

O ideal é que as sobras e medicamentos vencidos sejam levados às farmácias e Unidades Básicas de Saúde pela população, a fim de que sejam depositados e a logística reversa segura possa ser realizada (BUENO; WEBER; OLIVEIRA, 2009).

Estando a informação e sua falta posicionada em um ponto central do problema de descarte de medicamentos, e sendo as Unidades Básicas de Saúde centros de informação e disseminação dos medicamentos municipais, ainda que exista rede de farmácias, as ações mitigativas devem passar pela informação sistemática da população acerca do descarte nessas unidades. Da mesma forma, assumir o papel ativo na logística reversa dos medicamentos é uma das possibilidades para minorar os descartes indevidos.

Em uma proposição de ações mitigativas, a abordagem e orientação da população, no momento da entrega dos medicamentos ou mesmo na disseminação comunitária de pontos de coleta são alternativas de baixo ônus e alta eficiência.

Essas medidas têm o respaldo da consideração da educação informal como medida de longo prazo, segura e definitiva para problemas emergentes causados pela desinformação e pelos efeitos que ocasionam na vida humana (SCHAFRANSKI, 2009). Abordam, ainda, a educação como prática social e inserida nos contextos de demanda, logo, são medidas pertinentes e apropriadas para a aplicação na demandante realidade da população estudada.

Os achados no lixo comum no estudo depreendido são muito similares aos propagados achados em ambientes aquáticos e são coerentes com as substâncias mais comumente consumidas na cultura medicamentosa nacional e internacional, com a prevalência de fármacos como Ibuprofeno.

A média de descarte de medicamentos ao ano, por habitante, igualmente, se mostrou elevada. Como problema de saúde pública, além dos riscos ambientais e de contaminação das pessoas eventualmente expostas a esses descartes, há ainda o ônus do desperdício de medicamentos. Os riscos e agravantes ambientais de uma rotina implantada de descarte elevado de fármacos no ambiente foram identificados no município de análise, avalizados pela chancela de estudos que consideram o efeito geral e isolado de muitas dessas substâncias.

A variada disposição de fármacos oferece a possibilidade de combinações diversas, que podem ter ecotoxidade imprevisível e afetar os espaços de deposição de modo direto ou indireto, através de resíduos oriundos de lama contaminada pelos fármacos ou mesmo do uso do solo para fins diversos, entre outras frentes.

\section{Conclusão}

Conclui-se que o descarte de medicamentos no lixo comum da cidade de Terenos, Mato Grosso do Sul, é feito de forma aleatória e, no período investigado, a maioria dos medicamentos ou insumos farmacêuticos. Dos medicamentos descartados ou insumos farmacêuticos a classe dos antiinflamatórios prevaleceu, o que favorece a contaminação ambiental.

A deposição de fármacos se mostrou uma prática recorrente, inclusive, com o hábito do descarte de medicações antes do seu vencimento, um indicativo de culturas de prescrição ou de hábitos de consumo e de automedicação, possivelmente, problemáticos. 
Com isso, as ações educativas, como abordagem mitigativa, são as proposições mais viáveis, uma vez que a população desponta com um hábito sistemático de descarte, a fim de estruturar uma logística reversa eficiente no município.

Para esse fim, as Unidades Básicas de Saúde e farmácias do município podem funcionar como postos de coleta, porém um trabalho sistemático de informação e orientação dos usuários, bem como de políticas mais consistentes no controle da prescrição e trato com os medicamentos devem ser consideradas como parte do processo e como fatores capazes de minorar os potenciais problemas ambientais e coletivos das deposições indevidas.

\section{Agradecimentos}

A pesquisa foi realizada com apoio da Coordenação de Aperfeiçoamento de Pessoal de Nível Superior Brasil (CAPES) - Código de Financiamento 001 e, da Universidade Anhanguera-Uniderp. Ao CNPq, CPP e FUNADESP, pelo apoio financeiro e a Bolsa de Produtividade CNPq-2 e 1D.

\section{Referências}

ABAHUSSAIN, E.A.; BALL, D.E. Disposal of unwanted medicines from households in Kuwait. Pharm. World Sci., v. 29, n.4, p.368-373, 2007. doi: 10.1007/s11096-006-9082-y

ALI, I. et al. Chiral analysis of ibuprofen residues in water and sediment. Anal. Letters, v.42, n. 12, p.1747-1760, 2009. doi: $10.1080 / 00032710903060768$

ALMEIDA, G.B.S.; PAZ, E.P.A.; SILVA, G.A.D.A. Social representations of arterial hypertension and its care: a collective subject discourse. Acta Paul. Enferm., v.24, n.4, p.459-465, 2011. doi: $10.1590 / \mathrm{S} 0103-21002011000400003$

ALVARENGA, L.S.V.; NICOLETTI, M.A. Domestic discarding of medicines and some considerations about the current environment impact. Saúde-UNG, v. 4, n. 3, p.34-39, 2010.

ANKLEY, G. T. et al. Repeating history: Pharmaceuticals in the environment. Environ. Scie. Technol., v.41, n.24, p.8211-8217, 2007. doi: 10.1021/es072658j

AQUINO, D. S. Por que o uso racional de medicamentos deve ser uma prioridade? Ciênc. Saúde Coletiva, v.13, p.733-736, 2008. doi: $10.1590 / \mathrm{S} 1413-81232008000700023$

BOUND, J.P.; VOULVOULIS, N. Predicted and measured concentrations for selected pharmaceuticals in UK rivers: Implications for risk assessment. Water Res., v.40, n.15, p.28852892, 2006. doi: 10.1016/j.watres.2006.05.036

BRASIL. Resolução da Diretoria Colegiada No 306. Brasília: MS, 2004. Disponível em: https://bvsms.saude.gov.br/bvs/ saudelegis/anvisa/2004/res0306_07_12_2004.html. Acesso em: 20 set. 2019.

BRASIL. Conselho Nacional do Meio Ambiente. RESOLUÇÃO CONAMA $n^{\circ} 358$, de 29 de abril de 2005. Dispõe sobre o tratamento e a disposição final dos resíduos dos serviços de saúde e dá outras providências. Disponível em:

http://www.siam.mg.gov.br/sla/download.pdf?idNorma=5046. Acesso em: 20 set. 2019.

BROOKS, B.W. et al. Aquatic ecotoxicology of fluoxetine. Toxicol. Lett., v.142, n.3, p.169-183, 2003. doi: 10.1016/s03784274(03)00066-3
BUENO, C.S.; WEBER, D.; OLIVEIRA, K.R. Farmácia caseira e descarte de medicamentos no bairro Luiz Fogliatto do município de Ijuí - RS. Rev. Cienc. Farm. Bas. Aplic., v.30, n.2, p.203-210, 2009.

CARVALHO, E.V. et al. Aspectos legais e toxicológicos do descarte de medicamentos. Rev. Bras. Toxicol., v.22, n.1/2, p.1-8, 2009.

CLEUVERS, M. Aquatic ecotoxicity of pharmaceuticals including the assessment of combination effects. Toxicol. Lett., v.142, n.3, p.185-194, 2003. doi: 10.1016/s0378-4274(03)000687

COSTA, I.L.; PLETSCH, A.L.; TORRES, Y.R. Occurrence of antidepressant drugs in the environment: a review. Rev. Virtual Quim., v.6, n.5, p.1408-1431, 2014.

DAUGHTON, C.G. Cradle-to-cradle stewardship of drugs for minimizing disposition while promoting human health. II Drug disposal, waste reduction, and future directions. Environ Health Perspect, v. 111, p.775-785, 2003. doi: 10.1289/ehp.5948

EICKHOFF, P.; HEINECK, I.; SEIXAS, L.J. Gerenciamento e destinação final de medicamentos: uma discussão sobre o problema. Braz. J. Pharm., v.90, n.1, p.64-68, 2009.

FALQUETO, E.; KLIGERMAN, D.C.; ASSUMPÇÃO, R.F. How to do the correct discard of medicine residues? Ciênc. Saúde Coletiva, v.15, p.3283-3293, 2010. doi:10.1590/S141381232010000800034.

FENT, K.; WESTON, A.A.; CAMINADA, D. Ecotoxicology of human pharmaceuticals. Aquatic Toxicol., v.76, n.2, p.122-159, 2006. doi: 10.1016/j.aquatox.2005.09.009

FERNANDES, L.C.; PETROVICK, P.R. Os medicamentos na farmácia caseira. In: SCHENKEL, E.P. Cuidados com os medicamentos. Porto Alegre: UFRGS, 2004. p.39-42.

FLAHERTY, C. M.; DODSON, S. I. Effects of pharmaceuticals on Daphnia survival, growth, and reproduction. Chemosphere, v.61, n.2, p.200-207, 2005. doi: 0.1016/j.chemosphere.2005.02.016

GHISELLI, G.; JARDIM, W.F. Interferentes endócrinos no ambiente. Quim. Nova, v.30, n.3, p.695-706, 2007. doi: 10.1590/ S0100-40422007000300032

GLASSMEYER, S.T. et al. Disposal practices for unwanted residential medications in the United States. Environ. Int., v. 35, n. 3, p. 566-572, 2009. doi:10.1016/j.envint.2008.10.007

IBGE. Instituto Brasileiro de Geografia e Estatística. Disponível em: $<$ https://cidades.ibge.gov.br/brasil/go/santa-fe-de-goias/pesq uisa/23/25888? detalhes=true $>$. Acesso em: 16 out. 2019.

PRANDINI, F. L. et al. Gerenciamento integrado do lixo municipal. In: D'ALMEIDA, M. L. O.; VILHENA, A. Lixo municipal: manual de gerenciamento integrado. São Paulo: IPT/ CEMPRE, 2000.

JOÃO, W. DA S. J. Descarte de medicamentos. Pharm. Bras., v. 82, p.14-16, 2011.

MARTINEZ, J.L. Environmental pollution by antibiotics and by antibiotic resistance determinants. Environ.l Pollution, v.157, n.11, p.2893-2902, 2009. doi: 10.1016/j.envpol.2009.05.051

MELO, V. Descarte de medicamentos vencidos por usuários residentes na cidade de Internacional. In: CONGRESSO PAULISTA DE FARMACÊUTICOS, 14; SEMINÁRIO CRUZ, 6. São Paulo. Anais... São Paulo: Faculdade Oswaldo Cruz, 2005.

MIGLIORE, L.; COZZOLINO, S.; FIORI, M. Phytotoxicity to and uptake of enrofloxacin in crop plants. Chemosphere, v.52, n. 7, p.1233-1244, 2003. doi: 10.1016/S0045-6535(03)00272-8 
MORLEY, N.J. Environmental risk and toxicology of human and veterinary waste pharmaceutical exposure to wild aquatic hostparasite relationships. Environ. Toxicol. Pharmacol., v.27, n.2, p.161-175, 2009. doi: 10.1016/j.etap.2008.11.004

PASCOE, D.; KARNTANUT, W.; MÜLlER, C.T. Do pharmaceuticals affect freshwater invertebrates? A study with the cnidarian Hydra vulgaris. Chemosphere, v.51, n.6, p.521-528, 2003. doi:10.1016/S0045-6535(02)00860-3

PROENÇA, P.N.P. Resíduos de medicamentos: estudo de caso sobre comportamentos, atitudes e conhecimentos. Portugal: Universidade Aberta, 2011.

SANTOS, E.C.; FERREIRA, M.A. A indústria farmacêutica e a introdução de medicamentos genéricos no mercado brasileiro. Rev. Nexos Econôm., v.6, n.2, p.95, 2012.

SANTOS, L.H.M.L.M. et al. Ecotoxicological aspects related to the presence of pharmaceuticals in the aquatic environment. J. Hazardous Mat., v.175, n.1/3, p.45-95, 2010. doi: 10.1016/j. jhazmat.2009.10.100

SANTOS, Z.M.S.A. et al. Adesão do cliente hipertenso ao tratamento: análise com abordagem interdisciplinar. Texto Contexto Enferm., v.14, n.3, p.332-340, 2005.

SCHAFRANSKI, M.D. A educação e as transformações da sociedade. Public. UEPG: Ciênc. Hum. Ling. Letras Artes, v.13, n.2, p.101-112, 2005.

SERAFIM, E.O.P. et al. Qualidade dos medicamentos contendo dipirona encontrados nas residências de Araraquara e sua relação com a atenção farmacêutica. Braz. J. Pharm. Scie., v.43, n.1, p.127-135, 2007.
SILVA, C.M. Gerenciamento de resíduos sólidos gerados em laboratório de análises clínicas na cidade de Ribeirão Preto-SP. São Paulo: Universidade de São Paulo, 2008.

TERNES, T.A. et al. Behavior and occurrence of estrogens in municipal sewage treatment plants - I. Investigations in Germany, Canada and Brazil. Scie. Total Environ., v.225, n.1/2, p.81-90, 1999. doi: 10.1016/S0048-9697(98)00334-9

TESSARO, P.R.; ZANCANARO, V. Recolhimento e descarte dos medicamentos das farmácias caseiras no município de Caçador SC. Saúde e Meio Amb., v.2, n.1, p.118-128, 2013. doi:10.24302/ sma.v2i1.449

TRIBESS JUNIOR, A.; ZANCANARO, V. Descarte de medicamentos domiciliares e impacto ambiental: Conscientização da população no município de Caçador/SC. Rev. Extensão em Foco, v. 1, n. 1, p. 54-58, 2013.

UEDA, J. et al. Impacto ambiental do descarte de fármacos e estudo da conscientização da população a respeito do problema. Rev. Ciênc. Amb. On-Line, v.5, n.1, p.1-6, 2009.

VENTURA, K.S. Diagnóstico dos resíduos sólidos de serviços de saúde. Instituto de Pesquisa Econômica Aplicada, p. 62, 2012.

VETTORAZZI, K. M.; VENAZZI, K. F. Responsabilidade socioambiental dos produtores de medicamentos e farmácias sobre os resíduos sólidos de saúde: a logística reversa como possibilidade de coleta e correta destinação. Cascavel: FAECentro Universitário, 2008.

ZUCCATO, E. et al. Pharmaceuticals in the environment in Italy: Causes, occurrence, effects and control. Environ. Scie. Pollution Res., v.13, n.1, p.15-21, 2006. doi: 10.1065/espr2006.01.004 